IASSNS-AST 96/58

SISSA 166/96/EP

hep-ph xxxxxxx

November 1996

\title{
Constraints on Energy Independent Solutions of the Solar Neutrino Problem
}

\author{
P.I. Krastev ${ }^{a} *$ and S.T. Petcov ${ }^{b}$ \\ ${ }^{a}$ School of Natural Sciences, Institute for Advanced Study, \\ Princeton, NJ 08540 \\ ${ }^{b}$ Scuola Internazionale Superiore di Studi Avanzati, and \\ Istituto Nazionale di Fisica Nucleare, Sezione di Trieste, I-34013 Trieste, Italy
}

\begin{abstract}
We analyze the latest published solar neutrino data assuming an arbitrary neutrino oscillation/conversion mechanism suppresses the electron neutrino flux from the Sun independent of energy. For oscillations/transitions into active (sterile) neutrinos such mechanisms are ruled out at 99.96 (99.9997) \% C.L. assuming the standard solar model by Bahcall and Pinnsoneault '95 correctly predicts all solar neutrino fluxes within their estimated uncertainties. Even if one allows for ${ }^{8} \mathrm{~B}$ and ${ }^{7} \mathrm{Be}$ solar neutrino fluxes that are vastly different from the ones in contemporary standard solar models these mechanisms are strongly disfavored by the data.
\end{abstract}

*Also at: Institute of Nuclear Research and Nuclear Energy, Bulgarian Academy of Sciences, 1784 Sofia, Bulgaria. 


\section{INTRODUCTION}

The present solar neutrino data [1 4], are in significant disagreement with the most elaborate contemporary standard solar models (SSM) [5]7]. Theoretical uncertainties and experimental errors cannot account for this discrepancy and the data indicate that the solar $\nu_{e}$ flux is reduced with respect to its predicted value in the SSM. Assuming the electron neutrinos $\left(\nu_{e}\right)$ produced in the Sun and later detected in the solar neutrino detectors on Earth, travel unaltered between the points of production and detection, and using the luminosity constraint one finds that the inferred from the data reduction of the intermediate energy ${ }^{7} \mathrm{Be}$ neutrinos is much stronger than the reduction of both the low-energy $p p$ neutrinos, the flux of which actually appears to be increased by $10 \%$ at the best fit point, and the high-energy ${ }^{8} \mathrm{~B}$ neutrinos, the flux of which turns out to be lower by a factor of $\simeq 0.3[$ []]

Presently the only known way to explain the discrepancy between the predictions of the solar models and the experimental results is to resort to neutrino flavor conversion mechanisms (MSW [9], vacuum oscillations [10], etc.) which can provide an energy dependent suppression of the known solar $\nu_{e}$ fluxes $\left(p p,{ }^{7} \mathrm{Be},{ }^{8} \mathrm{~B}, p e p,{ }^{13} \mathrm{~N}\right.$ and $\left.{ }^{15} \mathrm{O}\right)$. An important role in the explanation plays also the $\nu_{\mu}$ (and/or $\nu_{\tau}$ ) neutral current contribution to the neutrino-electron scattering rate, present in the case of solar $\nu_{e}$ oscillations/transitions into active neutrinos. This contribution increases the ratio of the event rate in the Kamiokande detector to the event rate expected from the SSM without oscillations, with respect to the analogous ratio for the chlorine experiment, in accordance with the data.

In a previous publication [11] we have shown that neutrino conversion mechanisms which predict an energy independent suppression of the entire solar neutrino spectrum are ruled

\footnotetext{
*Here and in what follows we always have in mind solar neutrino conversion mechanisms which lead to an energy independent and equal suppression of all components of the solar $\nu_{e}$ flux. For brevity we call such mechanisms "energy independent mechanisms". Let us note that, for instance, the resonant solar neutrino transitions due to the interplay of neutrino flavour changing and flavour
} 
out by the data, assuming the standard solar model [13] correctly predicts all neutrino fluxes. We also showed that even if the ${ }^{8} \mathrm{~B}$ neutrino flux is reduced by one half and the ${ }^{7} \mathrm{Be}$ neutrino flux is reduced by $30 \%$ with respect to the corresponding theoretically predicted fluxes in the SSM, these mechanisms still provide a poor fit to the data. Examples of such mechanisms with energy independent suppression are neutrino spin-precession [14], vacuum oscillations with an oscillation length much shorter then the Sun - Earth distance, adiabatic MSW transitions for all neutrino flux components, possible only for large mixing angles and $10^{-7} \mathrm{eV}^{2} \leq \Delta m^{2} \leq 10^{-5} \mathrm{eV}^{2}$, etc.

Our previous analysis has been published as a part of a larger investigation which was centered on somewhat different problems, used older data and by now an outdated reference solar model. Here we update our previous results by using the latest solar neutrino data

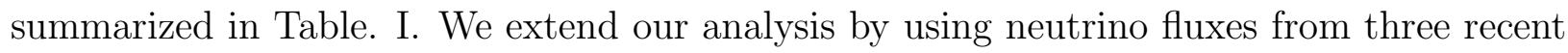
solar models [5-7] which include helium and heavy elements diffusion, an effect that has been shown lately to considerably improve the agreement with helioseismological very precise measurements of low degree p-modes [15,16]. We also use recently updated cross-sections for the chlorine and gallium detectors [17] which improve the accuracy of the predicted event rates in these detectors. We explain in detail the assumptions and numerical procedures used in the analysis. Our latest results confirm and strengthen the results and the conclusions reached in our previous publication [11].

\section{NUMERICAL PROCEDURE}

Given the published experimental results, a standard solar model which predicts the neutrino fluxes, and a mechanism depleting the fluxes of solar $\nu_{e}$ by a factor $P$ which

diagonal (but flavour dependent) neutral current interactions [12] are in the case of massless neutrinos energy independent. Nevertheless, the corresponding transition probabilities can be different for the ${ }^{8} \mathrm{~B},{ }^{7} \mathrm{Be}, p p$, etc. neutrinos (see ref. [12] for details). 
is independent of energy, one can compute $\chi^{2}$ including the theoretical uncertainties and experimental errors as described, e.g., in [18]. The theoretical uncertainties are due to uncertainties in the input parameters of the standard solar model as well as to uncertainties in the detection cross-sections. The correlations between the solar neutrino fluxes which arise because one and the same input parameter can affect several neutrino fluxes also have to be taken into account. In this calculation we have only one independent parameter, $P$, which is supplied by the relevant mechanism proposed as a solution of the solar neutrino problem. We describe the results of this calculation in the next section.

We next relax the uncertainty intervals, estimated within the SSM, of two of the most uncertain solar neutrino fluxes, ${ }^{8} \mathrm{~B}$ and ${ }^{7} \mathrm{Be}$. For three representative values of the beryllium neutrino flux, $\Phi_{B e}$, equal to $0.7,1.0$ and 1.3 times the flux in the SSM we vary both $P$ and the total boron neutrino flux, $\Phi_{B}$, treating them as free parameters [11]. This procedure was adopted in order to study how much model dependent are the strong constraints, obtained with the first procedure described above, on the energy independent neutrino oscillation/conversion mechanisms. One of the largest uncertainties of the solar model predictions is related to the fact that the cross-section for the ${ }^{7} \mathrm{Be}(p, \gamma){ }^{8} \mathrm{~B}$ reaction has never been measured at the typical energies at which this reaction is taking place in the Sun. This is the primary source of the large existing uncertainty in the predicted boron neutrino flux. In the model [0] the estimated one sigma relative uncertainty in the ${ }^{8} \mathrm{~B}$ flux is $\left({ }_{-0.17}^{+0.14}\right)$ and the corresponding uncertainty in the ${ }^{7} \mathrm{Be}$ neutrino flux is $\left({ }_{-0.07}^{+0.06}\right)$. The relative uncertainties in the SSM prediction of the CNO neutrino fluxes, ${ }^{13} \mathrm{~N}$ and ${ }^{15} \mathrm{O}$, are even larger than those of the boron flux. However, because of their lower energies and relatively low fluxes, the $\mathrm{CNO}$ neutrinos contribute much less to the event rates in the chlorine and gallium neutrino experiments than the ${ }^{8} \mathrm{~B}$ and/or ${ }^{7} \mathrm{Be}$ neutrinos and thus are less important in the analyses of the solar neutrino data. Therefore in our calculations we keep the CNO neutrino fluxes equal to their SSM values.

The predicted event rates in the operating detectors can be expressed as functions of the corresponding event rates as calculated from the SSM, the constant suppression factor, $P$, 
and the assumed ratios, $f_{\alpha}$, of each solar neutrino flux, $\Phi_{\alpha}$, to the same flux in the SSM [5], $\Phi_{\alpha, S S M}, f_{\alpha}=\Phi_{\alpha} / \Phi_{\alpha, S S M}, \alpha=p p,{ }^{7} \mathrm{Be}, p e p,{ }^{8} \mathrm{~B},{ }^{13} \mathrm{~N}$ and ${ }^{15} \mathrm{O}$. The three equations which give these event rates are:

$$
\begin{aligned}
Q_{G a} & =P\left(f_{B} Q_{G a}^{B}+f_{B e} Q_{G a}^{B e}+f_{p p} Q_{G a}^{p p}+f_{p e p} Q_{G a}^{p e p}+Q_{G a}(C N O)\right), \\
Q_{C l} & =P\left(f_{B} Q_{C l}^{B}+f_{B e} Q_{C l}^{B e}+f_{p e p} Q_{C l}^{p e p}+Q_{C l}(C N O)\right), \\
R_{\nu e} & =f_{B}\left(0.845 P_{B}+0.155\right), \\
\text { or } & \\
R_{\nu e} & =f_{B} P_{B} .
\end{aligned}
$$

The quantities in the lefthand side of the first two equations are the rates of ${ }^{71} \mathrm{Ge}$ and ${ }^{37} \mathrm{Ar}$ production in the gallium and chlorine detectors and $R_{\nu e}$ in Eq. (1d) and Eq. (1d) is the ratio (measured ${ }^{8} \mathrm{~B}$ flux)/(SSM ${ }^{8} \mathrm{~B}$ flux) for the Kamiokande $\left(\nu_{e} e\right)$ scattering experiment. Depending on the particular mechanism involved, $P$ can be the solar $\nu_{e}$ survival probability for neutrino oscillations, spin-flavor conversion, etc. For neutrino oscillations/transitions into active neutrinos $\left(\nu_{\mu}\right.$ and/or $\left.\nu_{\tau}\right)$, or antineutrinos $\left(\bar{\nu}_{\mu}\right.$ and/or $\left.\bar{\nu}_{\tau}\right)$, Eq. (10) is the relevant one, whereas Eq. (피) has to be used for oscillations/transitions into sterile neutrinos $\left(\nu_{s}\right)$. $Q_{i}^{\alpha}$ is the contribution of source $\alpha$ to the total event rate in the i-th radiochemical detector $(i=G a, C l)$ as predicted in the SSM. The coefficients $f_{\alpha}$ are written explicitly only for those fluxes for which we have assumed that they might be different from the ones predicted in the SSM.

For $f_{B e}=0.7(1.3)$ we chose to satisfy the luminosity constraint including small corrections for thermal motion of the particles in the plasma and inequalities between the neutrino fluxes, which follow from the sequencial process of nuclear fusion in the Sun [19] [1, by increasing (decreasing) the $p p$ neutrino flux by $\simeq 2 \%$. We always kept the ratio of the $p e p$ to $p p$ flux equal to the one in the SSM. The prediction for this ratio in the different solar

${ }^{\dagger}$ For earlier discussions of the luminosity constraint see ref. 20]. 
models is remarkably stable: it doesn't vary by more than one percent. The numerical coefficients in Eq. (IC) have been computed using the neutrino-electron scattering cross-sections with radiative corrections [21]. The ratio (0.155) of the (integrated over the ${ }^{8} \mathrm{~B}$ spectrum) scattering cross-sections $\sigma\left(\nu_{\mu}\left(\nu_{\tau}\right) e\right) / \sigma\left(\nu_{e} e\right)$ has been computed using the published trigger efficiency functions and energy resolution of the detector. We have taken into account also the known evolution of the trigger in Kamiokande [2]. During the first 449 days the detectors has been operated with a threshold of $9.3 \mathrm{MeV}$, later on the threshold has been reduced to $7.5 \mathrm{MeV}$ (794 days) and 7.0 MeV (836 days). A slight change in the trigger efficiency function during the Kamiokande-II stage due to increased light collection efficiency has been neglected. This effect might change the time-averaged ratio at most by a few percent, which will not change significantly any of our results.

Next we computed the $\chi^{2}$ defined as:

$$
\chi^{2}=\sum_{i=1}^{4} \frac{\left(Q_{e x p}(i)-Q_{t h}(i)\right)^{2}}{\sigma_{e x p}^{2}(i)+\sigma_{t h}^{2}}
$$

The index $i$ denotes the four operating experiments. The statistical and systematic errors from each experiment have been combined quadratically to find the total experimental error, $\sigma_{\exp }(i)$. The theoretical error is due to the uncertainties in the solar $\nu_{e}$ detection crosssections for the two radiochemical experiments [17] and has been calculated as described in [18]. The theoretical uncertainty for the Kamiokande experiment has been assumed to be zero. Here we neglected the theoretical uncertainties related to the input parameters in the solar model but instead varied two of the most uncertain fluxes, $\Phi_{B}$ and $\Phi_{B e}$, within much broader intervals than their estimated $3 \sigma$ uncertainty ranges in the SSM. This approach allows us to do the same analysis with three independently developed standard solar models [5] 0 .

\section{RESULTS}

Assuming the standard solar model [5] correctly predicts, within the estimated theoretical uncertainties, the flux from each solar neutrino source, the energy independent mechanisms 
provide a very poor fit to the data. For oscillations/transitions into active neutrinos the minimum $\chi^{2}$ is 13.0 ( 1 d.f.) which rules out this scenario at $99.96 \%$ C.L. The constraint on oscillations/transitions into sterile neutrinos are even stronger. The minimum $\chi^{2}$ is 23.5 which rules out this hypothesis at $99.9997 \%$ C.L. The reason for the difference between the C.L. at which oscillations/transitions into active and into sterile neutrinos are ruled out is the absence in the latter case of neutral current contribution to the neutrino-electron scattering rate which exacerbates the difficulty in describing simultaneously the Homestake and Kamiokande data.

The constraints given above depend on the solar model used in the analysis. Of the three recent solar models [5] [] only Ref. [5] gives a complete description of the estimated uncertainties in the neutrino fluxes related to the inaccurately known input parameters. In order to study this dependence on the solar model, as explained in the previous section, we disregard the solar model uncertainties of the most uncertain of the major solar neutrino fluxes, $\Phi_{B}$ and $\Phi_{B e}$. For $f_{B e}$ we chose three representative values, $f_{B e}=0.7,1.0$ and 1.3. In each of these three separate cases $f_{B}$ as well as $P$ are treated as free parameters. For each of the three solar models [5 [7] we computed the $\chi^{2}$ (Eq. 2) for a large number of pairs $\left(f_{B}, P\right)$ from the following intervals: $0 \leq f_{B} \leq 2$ and $0 \leq P \leq 1$, and found the minimum, $\chi_{\text {min }}^{2}$, for each of the three representative values of $f_{B e}$. The value of $\chi_{\min }^{2}$ determine the C.L. at which the tested hypotheses are ruled out by the data. The results are summarized in Table 【I] for the solar models [5-7] and for oscillations/transitions into active as well as into sterile neutrinos.

For $f_{\mathrm{Be}}=1$ the energy independent solar $\nu_{e}$ suppression mechanisms are ruled out at (96.5)\% C.L. $\left(\chi^{2}=6.8\right.$ (2 d.f.) $)$ for all three solar models studied here. Even if we relax the constraint on the ${ }^{7} \mathrm{Be}$ flux, the energy independent mechanisms still give a poor fit to the data. The smallest minimum $\chi_{\min }^{2}=5.9$ for oscillations/transitions into active neutrinos is achieved for $f_{B e}=0.7$. This rules out any energy independent mechanism as a solution of the solar neutrino problem at $94.5 \%$ C.L. (2 d.f.). Higher values of $f_{B e}$ are ruled out at even higher $\left(\simeq 97.5 \%\right.$ for $\left.f_{B e}=1.3\right)$ C.L. The constraints on neutrino oscillations/transitions 
into sterile neutrinos are somewhat stronger, e.g., for $f_{B e}=0.7$ the $\chi_{\min }^{2}$ is 8.2. This rules out such mechanisms at (at least) $98 \%$ C.L.

The week dependence of the results on the assumed beryllium neutrino flux shows that the residual theoretical uncertainties, which we have neglected here and which are related to all other neutrino fluxes except ${ }^{8} \mathrm{~B}$ and ${ }^{7} \mathrm{Be}$, cannot significantly change these results. Those uncertainties are either considerably smaller ( $p p$ and $p e p$ neutrinos) than the uncertainties in the ${ }^{8} \mathrm{~B}$ and ${ }^{7} \mathrm{Be}$ neutrino fluxes, which have been allowed to vary outside their estimated $3 \sigma$ limits, or are related to sources $(\mathrm{CNO})$, which give minor contributions to the event rates in the operating experiments. In several test cases we have verified this by a complete calculation where for the model [5], in addition to varying $f_{B}$ and $P$, we took into account the residual uncertainties in the $\mathrm{CNO}$, pep and $p p$ neutrino fluxes.

In our analysis we did not include solar models without diffusion, e.g., [22]. These models are strongly disfavored by helioseismological data [15, 16] although the predicted neutrino fluxes are only slightly lower than in the more advanced models included in Table [1]. We also did not include results for the model in ref. [23]. The results for this model, which differs from the models [5 [7] mainly by the choice of the astrophysical factors, give somewhat lower $\chi_{\min }^{2}$ in all cases changing the C.L. by less than $0.5 \sigma$.

The constraints so obtained on the energy independent solar $\nu_{e}$ suppression mechanisms depend only weakly on the particular solar model used as a reference. In addition, we want to emphasize that at present there is no compelling reason to expect such strong deviations, as the ones considered here, from the predicted fluxes in standard solar models. Our goal was to show that even ad hoc changes in the solar neutrino fluxes cannot avert our main result, namely that the neutrino oscillation/conversion mechanisms which predict energy independent suppression are strongly disfavored by the data from the operating solar neutrino experiments.

In order to test the significance of the data from each solar neutrino experiment for the poor fit provided by energy independent neutrino oscillations/transition mechanisms we have computed the $\chi^{2}$ for each of the three possible pairs of experiments, namely $(\mathrm{ClAr}+$ 
$(\nu e)),((\nu e)+\mathrm{GaGe})$ and $(\mathrm{GaGe}+\mathrm{ClAr})$. When only $P$ is allowed to vary and the neutrino fluxes are assumed to be the ones from the SSM in Ref. [5], the minimum $\chi^{2}$ for the three cases, assuming oscillations/transitions into active neutrinos, are:

$$
\begin{gathered}
\mathrm{ClAr}+(\nu e): \chi_{\text {min }}^{2}=0.07 ; \quad P=0.28, \\
(\nu e)+\mathrm{GaGe}: \chi_{\text {min }}^{2}=2.22 ; \quad P=0.50, \\
\mathrm{GaGe}+\mathrm{ClAr}: \chi_{\text {min }}^{2}=10.7 ; \quad P=0.46 .
\end{gathered}
$$

For oscillations/transitions into sterile neutrinos we obtain:

$$
\begin{gathered}
\mathrm{ClAr}+(\nu e): \chi_{\text {min }}^{2}=1.15 ; \quad P=0.34, \\
(\nu e)+\mathrm{GaGe}: \chi_{\text {min }}^{2}=0.79 ; \quad P=0.50, \\
\mathrm{GaGe}+\mathrm{ClAr}: \chi_{\text {min }}^{2}=10.7 ; \quad P=0.46 .
\end{gathered}
$$

From these results it is evident that (both for oscillations/transitions into active and sterile neutrinos) when only ( $\mathrm{GaGe}+\mathrm{ClAr})$ experiments are considered the energy independent solutions are strongly disfavored by the data whereas both $(\mathrm{ClAr}+(\nu e))$ and $(\mathrm{GaGe}+$ $(\nu e))$ data can be fitted by an energy independent suppression.

When both $P$ and the boron neutrino flux are allowed to vary for any pair of experimental results there is always an acceptably small $\chi_{m i n}^{2}$. The values of $P$ and $f_{B}$ for which $\chi^{2}$ is minimum are slightly different for the different solar models. However, the assumption that both the SSM and at least one of the solar neutrino experiments are wrong, seems too extreme and hardly justifiable.

Some energy independent mechanisms predict a fixed value of the survival probability which cannot be varied. Examples of such mechanisms are neutrino oscillations with maximal mixing between two $(\mathrm{P}=1 / 2)$ or three $(\mathrm{P}=1 / 3)$ neutrinos [24], as well as the mechanism discussed in ref. [25] $(\mathrm{P}=5 / 9)$. The results in Table III] show that all three values of the survival probability, $\mathrm{P}=1 / 3,1 / 2,5 / 9$, are ruled out by the data both for oscillations/transitions into active and into sterile neutrinos. The $\chi_{\min }^{2}$ values found correspond respectively to $99.4,96.5,94.5 \%$ C.L. and to 99.96, 99.4, $98.4 \%$ C.L. for the two 
types of oscillations/transitions. Not only are the minimum $\chi^{2}$ too high for all three models considered, but the values of the boron and especially of the beryllium neutrino fluxes, for which the C.L. is lowest, are far outside the acceptable uncertainty ranges for these fluxes.

\section{CONCLUSIONS}

Mechanisms proposed as solutions of the solar neutrino problem and predicting an energy independent suppression of the solar neutrino spectrum as a result of neutrino oscillations/transitions into active (sterile) neutrinos or antineutrinos are ruled out by the data from the four operating experiments 표 at 99.96 (99.9997) \% C.L., if the standard solar model [5] correctly predicts (within the estimated uncertainties) the solar neutrino fluxes from the different sources. Even relaxing the constraints on the ${ }^{7}$ Be neutrino flux by assuming that it differs by $30 \%$ from it's values in three recent solar models with heavy element diffusion, and treating the ${ }^{8} \mathrm{~B}$ neutrino flux as a free parameter, still provides a poor fit to the data for any value of the energy independent suppression factor. Suppression factors of $1 / 3,1 / 2$, and 5/9 24,25] are ruled out by the data at 99.4, 96.5, 94.5\% C.L. (99.96, 99.4,

$98.4 \%$ C.L.) for oscillations/transitions into active (sterile) neutrinos even if one treats both the ${ }^{8} \mathrm{~B}$ and ${ }^{7} \mathrm{Be}$ fluxes as free parameters in the analysis. Not only are the minimum $\chi^{2}$ too high, but the lowest $\chi_{\min }^{2}$ values are reached for boron and especially beryllium neutrino fluxes which lie far outside the acceptable uncertainty ranges for these fluxes.

\section{ACKNOWLEDGMENTS}

P.K. is thankfull to K. Babu and J.N. Bahcall for very usefull and stimulating discussions. The work of P.K. was partially supported by NSF grant \#PHY-9513835, while the work of S.T.P. was supported in part by the EEC grant ERBCHRX CT930132 and by grant PH-510 from the Bulgarian Science Foundation. 


\section{TABLES}

TABLE I. Solar neutrino data used in the analysis and the theoretical predictions from the SSM [5]. The units are SNU for the event rates in the radiochemical detectors and $\mathrm{cm}^{-2} \mathrm{~s}^{-1}$ for the flux measured by Kamiokande. For both the chlorine and gallium experiments the theoretically predicted event rates have been updated following the results in Ref. [17].

\begin{tabular}{lccc}
\hline \hline Experiment & Data & Theory & Units \\
\hline Chlorine & $2.56 \pm 0.16 \pm 0.14$ & $9.5_{-1.4}^{+1.2}$ & SNU \\
Kamiokande & $2.80 \pm 0.19 \pm 0.33$ & $6.62_{-1.12}^{+0.93}$ & $10^{6} \mathrm{~cm}^{-2} \mathrm{~s}^{-1}$ \\
GALLEX & $69.7 \pm 6.7_{-4.5}^{+3.9}$ & $136.8_{-7}^{+8}$ & $\mathrm{SNU}$ \\
SAGE & $72.0_{-10-7}^{+12+5}$ & $136.8_{-7}^{+8}$ & $\mathrm{SNU}$ \\
\hline \hline
\end{tabular}


TABLE II. Minimum $\chi^{2}$ for the energy independent mechanisms including oscillations/transitions into active or sterile neutrinos. The results are given for three reference solar models [5 7]. The ${ }^{7}$ Be neutrino flux has been chosen: a) equal to the one in the corresponding solar model, b) $30 \%$ higher and c) $30 \%$ lower. The values of $f_{B}$, the ratio of the boron neutrino flux to the predicted ${ }^{8} \mathrm{~B}$ flux in the relevant SSM and $P$, the energy independent suppression factor, for which $\chi_{\min }^{2}$ is achieved in each case, are given also.

\begin{tabular}{|c|c|c|c|c|c|c|c|}
\hline \multirow[b]{2}{*}{ solar model } & \multirow[b]{2}{*}{$f_{B e}$} & \multicolumn{3}{|c|}{ active } & \multicolumn{3}{|c|}{ sterile } \\
\hline & & $\chi_{\min }^{2}$ & $f_{B}$ & $P$ & $\chi_{\min }^{2}$ & $f_{B}$ & $P$ \\
\hline & 0.7 & 6.6 & 0.47 & 0.55 & 9.2 & 0.47 & 0.56 \\
\hline \multirow[t]{3}{*}{ BP'95 } & 1.0 & 7.5 & 0.51 & 0.49 & 10.7 & 0.49 & 0.51 \\
\hline & 1.3 & 8.2 & 0.54 & 0.45 & 12.0 & 0.51 & 0.47 \\
\hline & 0.7 & 5.9 & 0.61 & 0.57 & 8.2 & 0.60 & 0.58 \\
\hline \multirow[t]{3}{*}{ CDFLR } & 1.0 & 6.8 & 0.64 & 0.52 & 9.6 & 0.62 & 0.54 \\
\hline & 1.3 & 7.5 & 0.67 & 0.48 & 10.8 & 0.64 & 0.50 \\
\hline & 0.7 & 6.7 & 0.50 & 0.56 & 9.3 & 0.49 & 0.57 \\
\hline \multirow[t]{2}{*}{ RVCD } & 1.0 & 7.6 & 0.54 & 0.50 & 10.9 & 0.52 & 0.52 \\
\hline & 1.3 & 8.4 & 0.57 & 0.46 & 12.3 & 0.54 & 0.48 \\
\hline
\end{tabular}


TABLE III. Minimum $\chi^{2}$ and the corresponding values of $f_{B}$ and $f_{B e}$ for three characteristic values of $P$ (the solar $\nu_{e}$ survival probability) in specific models predicting energy independent suppression of the solar neutrino spectrum. The results are given for three solar models [5]7. In each case the upper row is for oscillations/transitions into active neutrinos, whereas the lower row is for oscillations transitions into sterile neutrinos.

\begin{tabular}{lccccccccc}
\hline \hline & \multicolumn{3}{c}{$\mathrm{P}=1 / 3$} & \multicolumn{3}{c}{$\mathrm{P}=1 / 2$} & \multicolumn{3}{c}{$\mathrm{P}=5 / 9$} \\
\cline { 2 - 9 } solar model & $\chi_{\text {min }}^{2}$ & $f_{B}$ & $f_{B e}$ & $\chi_{\text {min }}^{2}$ & $f_{B}$ & $f_{B e}$ & $\chi_{\min }^{2}$ & $f_{B}$ & $f_{B e}$ \\
\hline BP'95 & 10.3 & 0.71 & 2.0 & 7.0 & 0.53 & 0.75 & 6.0 & 0.50 & 0.44 \\
& 16.2 & 0.74 & 2.0 & 10.1 & 0.55 & 0.67 & 8.3 & 0.52 & 0.36 \\
\hline CDFLR & 11.1 & 0.97 & 2.0 & 6.8 & 0.69 & 0.91 & 5.8 & 0.65 & 0.55 \\
& 16.5 & 1.01 & 2.0 & 9.8 & 0.72 & 0.81 & 8.1 & 0.67 & 0.48 \\
\hline RVCD & 11.1 & 0.78 & 2.0 & 7.4 & 0.57 & 0.78 & 6.3 & 0.53 & 0.48 \\
& 17.2 & 0.81 & 2.0 & 10.6 & 0.59 & 0.70 & 8.8 & 0.55 & 0.40 \\
\hline \hline
\end{tabular}




\section{REFERENCES}

[1] K. Lande, Talk given at the Neutrino '96 Int. Conference, June 13 - 19, 1996, Helsinki, Finland (to be published in the Proceedings of the Conference); see also: R. Davis, Prog. Part. Nucl. Phys. 32, 13 (1994); B. T. Cleveland et al., Nucl. Phys. B (Proc. Suppl.) 38, 47 (1995).

[2] Y. Fukuda et al., (Kamiokande Collaboration), Phys. Rev. Lett. 77, (1996) 1683.

[3] T. Kirsten et al. (GALLEX Collaboration), Talk given at the Neutrino '96 Int. Conference, June 13 - 19, 1996, Helsinki, Finland (to be published in the Proceedings of the Conference); for earlier results see: P. Anselmann et al., Phys. Lett. B 327, 377 (1994); 342, 440 (1995); 357, 237 (1995).

[4] V. Gavrin et al. (SAGE Collaboration), Talk given at the Neutrino '96 Int. Conference, June 13 - 19, 1996, Helsinki, Finland (to be published in the Proceedings of the Conference); see also: G. Nico et al., in Proceedings of the XXVII International Conference on High Energy Physics, Glasgow, Scotland, 1994, edited by P. J. Busse y and I. G. Knowles (Institute of Physics, Bristol, 1995), p. 965; J. N. Abdurashitov et al., Phys. Lett. B 328, 234 (1994).

[5] J.N. Bahcall and M.H. Pinsonneault, Rev. Mod. Phys. 671 (1995); for a detailed description of the physics involved in solar modeling see J.N. Bahcall, Neutrino Astrophysics (Cambridge University Press, Cambridge, England, 1989).

[6] V. Castellani et al., preprint INFNE-10-96, astro-ph/9606180(to be published in Physics Reports (1996)).

[7] O. Richard, S. Vauclair, C. Charbonnel and W.A. Dziembowski, submitted to Astronomy and Astrophysics, (1996).

[8] J.N. Bahcall and M. Pinsonneault, "Status of Solar Models", to be published in the Proceedings of Neutrino '96, Helsinki, Finland, June 1996, hep-ph/9610542. 
[9] L. Wolfenstein, Phys. Rev. D 17 (1978) 2369; S.P. Mikheyev and A.Yu. Smirnov, Yad. Fiz. 42 (1985) 1441 (Sov. J. Nucl. Phys. 42 (1985) 913); Nuovo Cim 9C (1986) 17.

[10] B. Pontecorvo, Zh. Exp. Teor. Fiz 34 (1958) 247 (Sov. Phys. JETP 7 (1958) 172); Z. Maki, M. Nakagawa and S. Sakata, Prog. Theor. Phys. 28 (1962) 870.

[11] P.I. Krastev and S.T. Petcov, Phys. Rev. D53 (1996) 1665.

[12] M. Guzzo, A. Masiero and S.T. Petcov, Phys. Lett. B 271 (1991) 172.

[13] J.N. Bahcall and M.H. Pinsonneault, Rev. Mod. Phys. 64885 (1992)

[14] L.B. Okun, M.B. Voloshin and M.I. Vysotskii, Sov. J. Nucl. Phys. 44 (1986) 440 (Yad. Fiz. 44 (1986) 677).

[15] J. Christensen-Dalsgaard, Proceedings of the 4th Int. Topical Workshop on New Trends in Solar Neutrino Physics, May 2 - 4, 1996, Laboratori Nazionali del Gran Sasso, L'Aquila, Italy (eds. V. Berezinsky and G. Fiorentini, INFN, 1996), p. 35.

[16] J.N. Bahcall, M.H. Pinsonneault, S. Basu and J. Christensen-Dalsgaard and M. Pinsonneault, "Are Solar Models Reliable", astro-ph/9610250.

[17] J.N. Bahcall et al., Phys. Rev. C 54 (1996) 411.

[18] G.L. Fogli and E. Lisi, Astropart. Phys. 3 (1995) 185.

[19] J.N. Bahcall and P.I. Krastev, Phys. Rev. D 53 (1995) 4211.

[20] M. Spiro and D. Vignaud, Phys. Lett. B 242 (1990) 279; V. Castellani at al., Phys. Rev. D 50 (1994) 4749; N. Hata, S. Bludman and P. Langacker, Phys. Rev. D 49 (1994) 3622; S.T. Petcov, Nucl. Phys. B (Proc. Suppl.) 43 (1995) 12; S. Degl'Innocenti, G. Fiorentini and M. Lissia, Nucl. Phys. B (Proc. Suppl.) 43 (1995) 66.

[21] J. Bahcall, M. Kamionkowski and A. Sirlin, Phys. Rev. D 51 (1995) 6146.

[22] C. Turck-Chiéze and I. Lopez, Ap. J. 408 (1993) 347; G. Berthomieu, J. Provost, P. 
Morel and Y. Lebreton, Astron. and Astrophys. 268 (1993) 775; I.J. Sackmann, A.J. Boothroyd and W.A. Fowler, Ap. J. 360 (1990) 727; J.N. Bahcall and R. Ulrich, Rev. Mod. Phys. 60 (1988) 297.

[23] A. Dar and G. Shaviv, Ap. J. 468 (1996) 933.

[24] A. Acker, S. Pakvasa, J. Learned and T.J. Weiler, Phys. Lett. B 298 (1993) 149; R. Foot and R.R. Volkas, Phys. Rev. D 52 (1995) 6595 and references therein; E. TorrenteLujan, University of Bern report BUTP-96, hep-ph/9604218.

[25] P.F. Harrison, D.H. Perkins and W.G. Scott, Phys.Lett. B 349 (1995) 137 and B 374 (1996) 111. 